\title{
A New Prenylated Flavanone from the Root Bark of Morus
}

\author{
Seong Su Hong, ${ }^{\dagger \dagger}$ Sungeun Hong, ${ }^{\S}$ Hak Ju Lee, ${ }^{\#}$ Woongchon Mar,,${ }^{\S, *}$ and Dongho Lee ${ }^{\dagger, * *}$ \\ ${ }^{\dagger}$ College of Life Sciences and Biotechnology, Korea University, Seoul 136-713, Korea. "E-mail: dongholee@korea.ac.kr \\ *Natural Products Research Institute, Gyeonggi Institute of Science \& Technology Promotion, Suwon 443-270, Korea \\ ${ }^{\S}$ Natural Product Research Institute, College of Pharmacy, Seoul National University, Seoul 151-742, Korea \\ *E-mail: mars@snu.ac.kr \\ ${ }^{\#}$ Korea Forest Research Institute, Seoul 130-712, Korea \\ Received May 21, 2013, Accepted May 31, 2013
}

Key Words : Morus, Moraceae, Prenylated flavanone, Oxygen-glucose deprivation

Mori Cortex Radicis, the root bark of Morus species (Moraceae), has been used in Korean and Chinese folk medicine. It has been mainly used for the treatment of beriberi, constipation, cough, dizziness, tinnitus, and urinary incontinence. Its constituents have been shown for antimicrobial, antiviral, cardioprotective, hepatoprotective, hypolipidemic, hypouricemic, and neuroprotective activities. ${ }^{1-5}$ In addition, cyanidin glucose isolated from Morus alba L. showed neuroprotective effects on cerebral ischemic injury. ${ }^{6}$ A number of compounds including prenylated flavonoids, stilbenes, benzofurans, terpenes, alkaloids, and other phenolic compounds were previously reported as chemical constituents of this tree. ${ }^{7-18}$ In our previous studies, some arylbenzofurans and prenylated flavonoids were isolated from this plant, and some of these compounds showed protective effects against dopamine-, nitric oxide- and oxygen and glucose deprivation (OGD)-induced cell death in neuroblastoma SH-SY5Y cells. ${ }^{19-21}$ In a continuing effort to discover neuroprotective metabolites from this plant, a new prenylated flavanone, named morusinone (1) [5,7,2',4'-tetrahydroxy3',5'-di(3-hydroxy-3-methylbutyl)flavanone], together with two known arylbenzofuran derivatives, mulberrofuran $\mathrm{J}$ (2) and albafuran $\mathrm{C}(\mathbf{3})$ was isolated from the methanol extract of Mori Cortex Radicis (Figure 1). This paper deals with the isolation and structure elucidation of a new prenylated flavanone based on spectroscopic techniques including MS, 1D and 2D NMR, and the biological evaluation of the compounds isolated for protective effects against OGDinduced neuronal cell death.

Compound 1 was obtained as a brown amorphous powder. The molecular formula was determined to be $\mathrm{C}_{25} \mathrm{H}_{32} \mathrm{O}_{8}$ by HRESIMS for the $[\mathrm{M}-\mathrm{H}]^{-}$ion at $\mathrm{m} / z 459.2024$ (calcd for $\mathrm{C}_{25} \mathrm{H}_{31} \mathrm{O}_{8}, 459.2019$ ), possessing ten degrees of unsaturation in the molecule. The presence of a flavanone skeleton was evident from the UV $\left(\lambda_{\max } 287 \mathrm{~nm}\right),{ }^{1} \mathrm{H}-\left[\delta_{\mathrm{H}} 5.68(1 \mathrm{H}, \mathrm{dd}, J=\right.$ $13.0,2.5 \mathrm{~Hz}, \mathrm{H}-2), 3.26$ (1H, dd, $J=17.5,13.0 \mathrm{~Hz}, \mathrm{H}-3_{\mathrm{ax}}$ ), and $\left.2.57\left(1 \mathrm{H}, \mathrm{dd}, J=17.5,2.5 \mathrm{~Hz}, \mathrm{H}-3_{\mathrm{eq}}\right)\right]$ and ${ }^{13} \mathrm{C}-\left[\delta_{\mathrm{C}} 74.2\right.$ (C-2), 41.6 (C-3), and 196.9 (C-4)] NMR spectra (Table 1). In addition, the ${ }^{1} \mathrm{H}$ NMR spectrum of 1 (Table 1) displayed two aromatic proton signals at $\delta_{\mathrm{H}} 5.88(2 \mathrm{H}$, overlap, H-6 and $8)$, and an aromatic singlet proton signal at $\delta_{\mathrm{H}} 6.98(1 \mathrm{H}, \mathrm{s}$, H-6'), as well as four phenolic hydroxyl signals at $\delta_{\mathrm{H}} 12.2$ (5-OH), 10.8 (7-OH), $8.31\left(2^{\prime}-\mathrm{OH}\right)$ and 8.29 (4'-OH). Also, in its ${ }^{1} \mathrm{H}$ and ${ }^{13} \mathrm{C}$ NMR spectra (Table 1), characteristic signals for four methylenes $\left[\delta_{\mathrm{H}} 2.64(2 \mathrm{H}, \mathrm{m}, \mathrm{H}-1 "), 2.55\right.$ (2H, m, H-1"'), 1.58 (2H, m, H-2"'), and 1.53 (2H, m, H-2"),<smiles>CC(C)(O)CCc1cc(C2CC(=O)c3c(O)cc(O)cc3O2)c(O)c(CCC(C)(C)O)c1O</smiles><smiles>O=C(O)c1cc(O)cc(O)c1C1C=C(F)CC(c2ccc(O)cc2O)C1c1cc(O)cc(-c2cc3ccc(O)cc3o2)c1</smiles>

2<smiles>CC1=C[C@H](c2cc3cc(-c4cc(O)cc(O)c4)oc3cc2O)[C@H](C(=O)c2ccc(O)cc2O)[C@H](c2ccc(O)cc2O)C1</smiles>

3

Figure 1. Structures of compounds 1-3. 
Table 1. ${ }^{1} \mathrm{H}$ and ${ }^{13} \mathrm{C}$ NMR spectroscopic data of compound $\mathbf{1}^{a}$

\begin{tabular}{|c|c|c|c|}
\hline Position & $\delta_{\mathrm{H}}(J$ in $\mathrm{Hz})$ & $\delta_{\mathrm{C}}$ & $\mathrm{HMBC}(\mathrm{H} \rightarrow \mathrm{C})$ \\
\hline 2 & $5.68 \mathrm{dd}(13.0,2.5)$ & $74.2 \mathrm{~s}$ & 4 \\
\hline \multirow[t]{2}{*}{3} & $3.26 \mathrm{dd}(17.5,13.0)$ & $41.6 \mathrm{~s}$ & $2,4,1^{\prime}$ \\
\hline & $2.57 \mathrm{dd}(17.5,2.5)$ & & 4 \\
\hline 4 & & $196.9 \mathrm{~s}$ & \\
\hline $4 a$ & & $101.7 \mathrm{~s}$ & \\
\hline 5 & & $163.5 \mathrm{~s}$ & \\
\hline 6 & $5.88^{b}$ & $95.7 \mathrm{~d}$ & $4 a, 5,7,8$ \\
\hline 7 & & $166.6 \mathrm{~s}$ & \\
\hline 8 & $5.88^{b}$ & $95.0 \mathrm{~d}$ & $4 a, 6,7,8 a$ \\
\hline $8 \mathrm{a}$ & & $163.5 \mathrm{~s}$ & \\
\hline $1^{\prime}$ & & $117.2 \mathrm{~s}$ & \\
\hline $2^{\prime}$ & & $150.5 \mathrm{~s}$ & \\
\hline $3^{\prime}$ & & $118.5 \mathrm{~s}$ & \\
\hline $4^{\prime}$ & & $153.3 \mathrm{~s}$ & \\
\hline $5^{\prime}$ & & $121.7 \mathrm{~s}$ & \\
\hline $6^{\prime}$ & $6.98 \mathrm{~s}$ & $124.5 \mathrm{~d}$ & $2,4^{\prime}, 1^{\prime \prime \prime}$ \\
\hline $1 "$ & $2.64 \mathrm{~m}$ & $18.4 \mathrm{t}$ & $2^{\prime}, 3^{\prime}, 4^{\prime}, 2^{\prime \prime}, 3^{\prime \prime}$ \\
\hline $2^{\prime \prime}$ & $1.53 \mathrm{~m}$ & $42.5 \mathrm{t}$ & 3', 1", 3", 4", 5" \\
\hline $3 "$ & & $69.4 \mathrm{~s}$ & \\
\hline 4" & $1.14 \mathrm{~s}$ & $29.3 \mathrm{q}$ & $2^{\prime \prime}, 3 ", 5^{\prime \prime}$ \\
\hline $5^{\prime \prime}$ & $1.14 \mathrm{~s}$ & $29.3 \mathrm{q}$ & 2", 3", 4" \\
\hline $1 " '$ & $2.55 \mathrm{~m}$ & $24.6 \mathrm{t}$ & 4', 5', 6', 2"', 3"' \\
\hline $2^{\prime \prime \prime}$ & $1.58 \mathrm{~m}$ & $44.0 \mathrm{t}$ & 5', 1"', 3"', 4"', 5"' \\
\hline $3^{\prime \prime \prime}$ & & $69.0 \mathrm{~s}$ & \\
\hline 4"' & $1.13 \mathrm{~s}$ & $29.3 \mathrm{q}$ & 2"', 3"', 5"' \\
\hline $5 " '$ & $1.13 \mathrm{~s}$ & $29.2 \mathrm{q}$ & 2"', 3"', 4"' \\
\hline $5-\mathrm{OH}$ & $12.2 \mathrm{~s}$ & & $4 a, 6,5$ \\
\hline $7-\mathrm{OH}$ & $10.8 \mathrm{~s}$ & & $6,7,8$ \\
\hline 2'-OH & $8.31 \mathrm{~s}$ & & $1^{\prime}, 2^{\prime}, 3^{\prime}$ \\
\hline 4'-OH & $8.29 \mathrm{~s}$ & & $3^{\prime}, 4^{\prime}, 5^{\prime}$ \\
\hline
\end{tabular}

${ }^{a}$ Measured at 500 and $125 \mathrm{MHz}$; obtained in DMSO- $d_{6}$ with TMS as an internal standard. The assignments were based on ${ }^{1} \mathrm{H}-{ }^{1} \mathrm{H}$ COSY, HSQC, and $\mathrm{HMBC}$ experiments. ${ }^{b}$ Overlapping signals

and $\delta_{\mathrm{C}} 44.0$ (C-2"'), 42.5 (C-2"), 24.6 (C-1"'), and 18.4 (C$1 ")]$, two gem-dimethyls [ $\delta_{\mathrm{H}} 1.14(6 \mathrm{H}, \mathrm{s}, \mathrm{H}-4 "$ and $5 ")$ and $1.13\left(6 \mathrm{H}, \mathrm{s}, \mathrm{H}-4{ }^{\prime \prime}\right.$ and 5"'), and $\delta_{\mathrm{C}} 29.3$ (C-4", 5", and 4"') and $\left.29.2\left(\mathrm{C}-5^{\prime \prime \prime}\right)\right]$, and two oxygenated quaternary carbons $\left[\delta_{\mathrm{C}} 69.4\left(\mathrm{C}-3^{\prime \prime}\right)\right.$ and $\left.69.0\left(\mathrm{C}-3^{\prime \prime \prime}\right)\right]$ were observed and these signals were suggestive of the presence of two 3-hydroxy-3methylbutyl moieties, which were substantiated by 2D NMR experiments. These results clearly indicated that compound 1 has a flavanone skeleton bearing two 3-hydroxy-3-methylbutyl groups. The positions of each substituents were determined unambiguously as C-5, C-7, C-2' and C-4' (four phenolic hydroxyls), and C-3' and C-5' (two 3-hydroxy-3methylbutyls), respectively, using HMBC NMR technique (Figure 2). Compound 1 did not show substantial optical activity, $[\alpha]_{\mathrm{D}}^{25}-1.1(c 0.11, \mathrm{MeOH})$, or Cotton effects in circular dichroism (CD). Therefore, the structure of this new compound was determined as 5,7,2',4'-tetrahydroxy-3',5'di(3-hydroxy-3-methylbutyl)flavanone, morusinone (1). In addition, two compounds of previously known structures were identified as mulberrofuran $\mathrm{J}(\mathbf{2})$ and albafuran $\mathrm{C}(\mathbf{3})$,

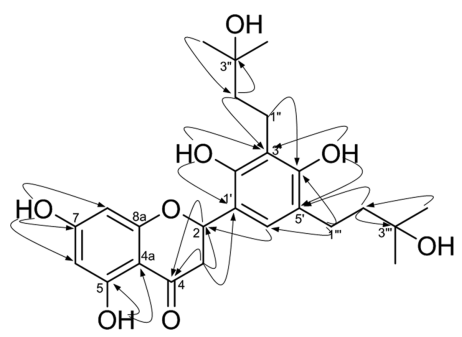

Figure 2. Key HMBC correlations of compound 1.

by spectral data interpretation and comparison with literature values. $^{22-24}$

Compounds 1-3 were tested for protective effects against OGD-induced cell death in neuroblastoma SH-SY5Y cells by using MTT reduction assay. The assay was performed according to established protocols. ${ }^{25-27}$ Morusinone (1) moderately enhanced neuronal cell viability under OGD and reoxygenation conditions in a dose-dependent manner with an $\mathrm{EC}_{50}$ value of $24.5 \mu \mathrm{g} / \mathrm{mL}\left(\mathrm{EC}_{50}\right.$ value of carnosine, a positive control, $8.4 \mu \mathrm{g} / \mathrm{mL}$ ), whereas compounds $\mathbf{2}$ and $\mathbf{3}$, at concentration up to $50 \mu \mathrm{g} / \mathrm{mL}$, were inactive in the assay.

\section{Experimental Section}

General Procedures. The optical rotations were measured using a JASCO DIP-1000 polarimeter. CD spectra were recorded on a JASCO J-715 spectrometer. UV spectra were obtained on a Mecasys Optizen 2120UV. NMR spectra were taken on a Varian $500 \mathrm{MHz}$ NMR spectrometer using tetramethylsilane (TMS) as an internal standard, and chemical shifts were expressed in $\delta$ values. Mass spectra were obtained on a Waters Q-TOF micro mass spectrometer. Column chromatography was performed using a silica gel (Kieselgel 60, 70-230 mesh, Merck), RP-18 (12 nm, S-75 $\mu \mathrm{m}$, YMC), and Sephadex LH-20 (18-111 mm, GE Healthcare), and thin layer chromatography was performed using a pre-coated silica gel $60 \mathrm{~F}_{254}(0.25 \mathrm{~mm}$, Merck). Preparative HPLC was conducted using a Varian Prostar 210 system.

Plant Material. The dry material of Mori Cortex Radicis was purchased from Kyungdong Oriental Herbal Market, Seoul, Korea, on August 2006 and identified by one of the authors (H. J. L.). A voucher specimen (20060811-MCR) has been deposited at College of Life Sciences and Biotechnology, Korea University, Korea.

Extraction and Isolation. The dry material of Mori Cortex Radicis $(10 \mathrm{~kg})$ was extracted three times with $\mathrm{MeOH}$ at room temperature. Removal of the solvent in vacuo yielded an $\mathrm{MeOH}$ extract $(1.5 \mathrm{~kg})$. The $\mathrm{MeOH}$ extract was suspended in distilled water and then partitioned with $n$-hexane and EtOAc (610 g), successively. Column chromatography of the EtOAc-soluble layer (75 g) over silica gel using a $\mathrm{CHCl}_{3}$ $\mathrm{MeOH}$ mixture with increasing polarity, yielded 12 fractions (SS2-1 12). Fraction SS2-7 was subjected to vacuum liquid chromatography on RP-18 eluting with $\mathrm{MeCN}-\mathrm{H}_{2} \mathrm{O}$ (20:80 to 100:0) to give nine fractions (SS2-6-1 9). Among the above nine fractions, compounds $1(10 \mathrm{mg})$ was obtained by 
an additional purification step on the RP-HPLC from fraction SS2-6-3. Fraction SS2-10 was subjected to flash column chromatography on RP-18 developing with MeCN$\mathrm{H}_{2} \mathrm{O}(50: 50)$ to give five fractions (SS2-7-1 5). Compounds $\mathbf{2}(38 \mathrm{mg})$ and $\mathbf{3}(42 \mathrm{mg})$ were isolated from fraction SS2-7-3 by column chromatography on a Sephadex LH-20 using $\mathrm{CHCl}_{3}-\mathrm{MeOH}(1: 1, \mathrm{v} / \mathrm{v})$.

Bioassay. Cell culture, oxygen and glucose deprivation followed by reoxygenation, and measurement of cellular viability by MTT assays were performed as described previously. $^{19}$

Morusinone (1) [5,7,2',4'-tetrahydroxy-3',5'-di(3-hydroxy-3-methylbutyl)flavanone]. Brown amorphous powder; $[\alpha]_{\mathrm{D}}^{25}-1.1(c 0.11, \mathrm{MeOH}) ; \mathrm{UV}(\mathrm{MeOH}) \lambda_{\max }(\log \varepsilon) 228$ (4.15), 287 (4.03), 339 (sh, 3.71) nm; ${ }^{1} \mathrm{H}$ and ${ }^{13} \mathrm{C}$ NMR (500 and $125 \mathrm{MHz}$ ), see Table 1; ESIMS $m / z 459$ [M - H] $]^{-}, 919$ $[2 \mathrm{M}-\mathrm{H}]^{-}$; HRESIMS (negative mode) $\mathrm{m} / z$ 459.2024 (calcd for $\mathrm{C}_{25} \mathrm{H}_{31} \mathrm{O}_{8}, 459.2019$ ).

Mulberrofuran J (2). Reddish powder; $[\alpha]_{\mathrm{D}}^{25}-101.3(c$ $0.1, \mathrm{MeOH})$; UV, MS, and NMR spectral data, see Supporting Information.

Albafuran C (3). Reddish powder; $[\alpha]_{\mathrm{D}}^{25}-76.5$ (c 0.13 , $\mathrm{MeOH}) ; \mathrm{UV}, \mathrm{MS}$, and NMR spectral data, see Supporting Information.

Acknowledgments. This study was supported by a grant from the National Forest Service, Korea (2012). This work was also funded by a Korea University Grant.

Supporting Information. The UV, MS, and NMR spectral data of compounds $\mathbf{2}$ and $\mathbf{3}$ are available as Supporting Information.

\section{References}

1. Park, K. M.; You, J. S.; Lee, H. Y.; Baek, N. I.; Hwang, J. K. J. Ethnopharmacol. 2003, 84, 181

2. Du, J.; He, Z. D.; Jiang, R. W.; Ye, W. C.; Xu, H. X.; But, P. P. Phytochemistry 2003, 62, 1235.

3. El-Beshbishy, H. A.; Singab, A. N.; Sinkkonen, J.; Pihlaja, K. Life
Sci. 2006, 78, 2724

4. Oh, H.; Ko, E. K.; Jun, J. Y.; Oh, M. H.; Park, S. U.; Kang, K. H.; Lee, H. S.; Kim, Y. C. Planta Med. 2002, 68, 932.

5. Park, W. J.; Lee, H. J.; Yang, S. G. Yakhak Hoeji 1990, 34, 434.

6. Kang, T. H.; Hur, J. Y.; Kim, H. B.; Ryu, J. H.; Kim, S. Y. Neurosci Lett. 2006, 391, 122.

7. Zhang, M.; Wang, R. R.; Chen, M.; Zhang, H. Q.; Sun, S.; Zhang, L. Y. Chin. J. Nat. Med. 2009, 7, 105.

8. Qiu, F.; Komatsu, K.; Kawasaki, K.; Saito, K.; Yao, X.; Kano, Y. Planta Med. 1996, 62, 559.

9. Hano, Y.; Kohno, H.; Suzuki, S.; Nomura, T. Heterocycles 1986, $24,2285$.

10. Hano, Y.; Nomura, T. Heterocycles 1986, 24, 1381.

11. Hano, Y.; Suzuki, S.; Nomura, T.; Ueda, S. Heterocycles 1989, 29 , 807.

12. Hano, Y.; Nomura, T.; Ueda, S. Heterocycles 1989, 29, 2035.

13. Nomura, T.; Fukai, T.; Matsumoto, J.; Ohmori, T. Planta Med. 1982, 46, 28.

14. Lee, S. H.; Choi, S. Y.; Kim, H.; Hwang, J. S.; Lee, B. G.; Gao, J. J.; Kim, S. Y. Biol. Pharm. Bull. 2002, 25, 1045.

15. Ueda, S.; Nomura, T.; Fukai, T.; Matsumoto, J. Chem. Pharm. Bull. 1982, 30, 3042 .

16. Ueda, S.; Matsumoto, J.; Nomura, T. Chem. Pharm. Bull. 1984, 32,350 .

17. Kusano, G.; Orihara, S.; Tsukamoto, D.; Shibano, M.; Coskun, M.; Guvenc, A.; Erdurak, C. S. Chem. Pharm. Bull. 2002, 50, 185.

18. Asano, N.; Yamashita, T.; Yasuda, K.; Ikeda, K.; Kizu, H.; Kameda, Y.; Kato, A.; Nash, R. J.; Lee, H. S.; Ryu, K. S. J. Agric. Food Chem. 2001, 49, 4208 .

19. Lee, H. J.; Lyu, D. H.; Koo, U.; Lee, S. J.; Hong, S. S.; Kim, K.; Kim, K. H.; Lee, D.; Mar, W. Arch. Pharm. Res. 2011, 34, 1373.

20. Lee, H. J.; Lyu, D. H.; Koo, U.; Nam, K. W.; Hong, S. S.; Kim, K.; Kim, K. H.; Lee, D.; Mar, W. Arch. Pharm. Res. 2012, 35 163.

21. Ham, A.; Lee, H. J.; Hong, S. S.; Lee, D.; Mar, W. Phytother. Res. 2012, 26, 620 .

22. Hirakura, K.; Hano, Y.; Fukai, T.; Nomura, T.; Uzawa, J.; Fukushima, K. Chem. Pharm. Bull. 1985, 33, 1088.

23. Takasugi, M.; Ishikawa, S.; Nagao, S.; Masamune, T. Chem. Lett 1982, 1223.

24. Dai, S. J.; Mi, Z. M.; Ma, Z. B.; Li, S.; Chen, R. Y.; Yu, D. Q. Planta Med. 2004, 70, 758

25. Goldberg, M. P.; Choi, D. W. J. Neurosci. 1993, 13, 3510.

26. Tabakman, R.; Jiang, H.; Shahar, I.; Arien-Zakay, H.; Levine, R. A.; Lazarovici, P. Ann. N. Y. Acad. Sci. 2005, 59, 1053.

27. Miglio, G.; Varsaldi, F.; Francioli, E.; Battaglia, A.; Canonico, P. L.; Lombardi, G. Eur. J. Pharmacol. 2004, 489, 157. 